\title{
Adherencia a la Dieta Mediterránea, Actividad Física y su relación con el Nivel Socioeconómico en escolares de Primaria de la capital de Granada The relationship of Mediterranean diet adherence and Physical Activity engagement with Socioeconomic Status in primary schoolchildren in the capital of Granada
}

Laura GarcíaPérez, Gracia CristinaVillodres, Francisco José M olina-Sánchez, Eva María Sánchez Cara, José J oaquín Muros M olina

Universidad de Granada (España)

\begin{abstract}
Resumen. En la adolescencia temprana se dan numerosas transformaciones y la adquisición de un estilo de vida saludable es trascendental para disminuir la aparición de enfermedades no transmisibles. 0 bjetivo: anal izar la relación entre la práctica de la actividad física (AF), la adherencia a la dieta mediterránea (DM) y el nivel socioeconómico (NSE). Material y métodos: en esta investigación de diseño transversal participaron 112 escolares del tercer ciclo de Educación Primaria de la capital de Granada, recogiendo los datos a través de tres cuestionarios: PAQ -C, para medir la práctica deAF; KIDM ED para medir la adherencia a la DM y FAS III para determinar el nivel socioeconómico familiar (NSE). Resultados: el $80 \%$ de la muestra necesita mejorar su alimentación, ya que solo dos de cada 10 niños presenta una alta adherencia a la DM, obteniendo puntuaciones ligeramente más altas el grupo masculino. Los niños practicaron másAF que las niñas. Por otro lado, se reveló que la condición socioeconómica es un factor influyente en la práctica de laAF, siendo el NSE bajo el grupo más desfavorecido en este parámetro. Por último, correlacionó positivamentela prácticadeAF con laadherenciaalaD M y el nivel socioeconómico. Conclusión: se denota la importancia de fomentar y mejorar tanto la práctica de AF como la adherencia al patrón dietético M editerráneo, desde edades tempranas. Resulta de vital importancia la creación de programas de intervención que fomenten la adquisición de hábitos saludables y busquen el equilibrio entre las desigualdades socioeconómicas del alumnado.
\end{abstract}

Palabras clave: Dieta M editerránea, Actividad física, Nivel socioeconómico, Escolares, Educación Primaria.

\begin{abstract}
A number of transitionsoccur during early adolescence that make the acquisition of heal thy lifestylestranscendental for decreasing the onset of noncommunicable diseases. 0 bjective: to analyse the relationship between the physical activity engagement (PA), Mediterranean diet adherence (MD) and socioeconomic status (SES). Material and methods: a crosssectional research study was conducted involving 112 schoolchildren enrolled on the third year of primary education in Granada. Data was collected data through three questionnaires. The PAQ -C estimated physical activity engagement, the KIDMED estimated M editerranean diet adherence and the FASIII determined family socioeconomic status. Results: $80 \%$ of participants need to improvetheir diet as only one fifth of participating children presented high M editerranean diet adherence. Slightly higher scores were obtained in the male group, with males also engaging in more physical activity than females. Further, it was reveal ed that socioeconomic status is an influential factor in relation to physical activity engagement, with those with the lowest socioeconomic status having the least favourable indices for this parameter. Finally, physical activity engagement was positively correlated with M editerranean diet adherence and socioeconomic status. Conclusion: present research highlights the importance of promoting and improving both PA engagement and adherence to M editerranean dietary patterns from an early age. The development of intervention programmes that promote the acquisition of healthy habits and seek a balance between the socio-economic inequalities among students.
\end{abstract}

Key Words: Mediterranean diet, Physical activity, Socioeconomic status, Schoolchildren, Elementary Education.

\section{Introducción}

Los factores relacionados con la promoción de hábitos saludables, como la práctica de actividad física (AF) o una buena adherencia a la dieta mediterránea (DM), sigue siendo de gran interés para la investigación edu-

Fecha recepción: 20-11-20. Fecha de aceptación: 14-01-21 Jose Joaquin Muros Molina

jjmuros@ugr.es cativa; debido a la repercusión positiva que tiene en los estilos de vida de los niños y niñas (Rodríguez, Iglesias \& Molina, 2020). El periodo de la preadolescencia se considera una etapa crítica debido a que, durante ella, se originan numerosas transformaciones a nivel físico, emocional, familiar y social (Patton \& Vinner, 2007); siendo esencial el desarrollo de hábitos y prácticas saludables que perduren a lo largo de toda la vida (Sánchez, Reyes \& González, 2014) y así evitar la aparición de 
problemas de salud en la edad adulta (GarridoFernández, García-Padilla, Sánchez-Ramos, Gómez Sal gado, Travé-González \& Sosa-Cordobés, 2020).

La actividad física (AF) se relaciona con diversos beneficios saludables a nivel físico y mental (Watson, Imperio, Brown, Best \& Hesketh, 2017) pero en general, los/ as niños/ as no ponen en práctica las recomendaciones deAF propuestas por la 0 rganización M undial de la Salud (O MS) (Guthol, Stevens, Riley \& Bull, 2020) ya que en 2010, a nivel mundial, el nivel de sedentarismo llegó a ser del $81 \%$ en la adolescencia (OMS,2007), siendo las chicas más inactivas que los chicos (López, Ahmed, López, Díaz \& Díaz, 2016). A pesar de haberse encontrado, en 2016, una leve mejora en la tendencia de la práctica deAF, solo para el caso del sexo masculino; no se considera suficiente para alcanzar una de las metas propuestas en la Asamblea Mundial de la Salud de 2018, cuyo objetivo eralareducción relativadel $15 \%$ de la inactividad física para el 2030 (OMS, 2019). En España, según los resultados de 2017 de la Encuesta Nacional deSalud (Instituto Nacional deEstadística INE, 2017), el $39.7 \%$ de la población infantil (de cero a 14 años) y el 30.2\% dela población joven (de 15 a 24 años) se declaró sedentaria. Esta ausencia de AF aumenta la probabilidad de padecer enfermedades cardiovasculares, obesidad, cáncer, diabetes, llegando a ser uno de los principales factores de riesgo de muerte a nivel mundial (OMS, 2017).

La adquisición de conductas saludables y su perdura ción en el tiempo no depende únicamente de la práctica de ejercicio físico, sino también de la adherencia a hábitos alimenticios adecuados (Tapia, 2019), ya que la adherencia a la dieta mediterránea (DM) también ha demostrado beneficios sobre factores de riesgo de estas enfermedades (Rees, Takeda, M artin, Ellis\&W ijesekara, 2019). Sin embargo, el perfil alimentario de la pobla ción española infantil se sitúa cada vez más alejado de la DM tradicional, principalmente por la insuficiencia de consumo de frutas, verduras y cereales de origen integral (González, García, Martos, Liébana \& M elo, 2015) tal y como lo indica el INE (2017) cuyas últimas cifras en 2017 registran que solo un $28.8 \%$ de la población de 5 a 14 años declara consumir verduras, ensaladas y hortalizas a diario así como solo un $57.6 \%$ de ellos, ingiere fruta fresca a diario. Estudios precedentes centrados en la adherencia a la DM, determinan la relación positiva entre el grado de adherencia a la DM y el nivel de AF realizada (Garrido-Fernández et al. , 2020; M uros, Cofre-Bolados, Arriscado, Zurita \& Knox, 2017; Farajian, Risvas, Karasouli, Pounis, Kastorini, Panagiotakos \&
Zampelas, 2011); por lo que, a mayores niveles de sedentarismo en edades tempranas, menor adherencia a la DM (Kontogianni et al., 2018).

La vinculación de práctica de ejercicio físico y adherencia a la DM, Ilega a ser un estilo de vida contextualizada tanto por factores geográficos y socioculturales (Ayechu \& Durà, 2009; Lahoz, Gracia \& Hervás, 2010); como por la condición socioeconómica; siendo esta una de las mayores influencias en el desarroIlo de los niños (Herrera-M ora, Munar-Torres, M olina Achury \& Robayo-Torres, 2019). El análisis del nivel socioeconómico (NSE) de la población es un aspecto primordial en el ámbito de la salud, condicionando el acceso a los servicios de la salud y en los resultados de la misma (Díaz-A costa Shiba-M atsumoto \& Gutiérrez, 2015). Además, las desigual dades socioeconómicas pueden ser una de las causas que modifiquen los patrones alimenticiosy actividad física (Sánchez-U rrea \& Izquierdo, 2021, p.95); ya que existen evidencias literarias que asocian directamente el estatus socioeconómico familiar con la calidad de dieta al imentaria y con la práctica deAF (Serra, Ribas, Pérez, Román \& Aranceta, 2003). Hay evidencias de que el NSE medio y alto muestra una mayor adherencia a la DM (Yannakoulia et al., 2016) y que los niños de familias con mayores recursos económicossuelen ser más activosy practicar másAF (Román, Serra, Ribas, Pérez-Rodrigo \& Aranceta, 2006).

Por lo anteriormente mencionado, el objetivo del estudio fue estudiar la relación entre diferentes variables: AF, adherencia a la DM y NSE, en escolares de tercer ciclo de Educación Primaria de la capital de Gra nada.

\section{Material y métodos}

\section{Participantes}

Se diseñó un estudio de carácter descriptivo y de corte transversal con una muestra de 112 escolares de 50 (87 alumnos) y 60 grado (25 alumnos) de Educación Primaria de la capital de Granada (España); correspondiendo a un rango de edad entre los 10 y 12 años (11.10 \pm 0.56$)$. De la muestra total, 64 eran niños (57.1\%) y 48 niñas (41.9\%).

\section{Instrumentos}

\section{Actividad física}

Para evaluar la práctica física se utilizó el cuestiona rio Physical Activity Questionnaire for older Children (PAQ C), utilizando su adaptación al castellano, traducción, adaptación transcultural y su validación (Martínez- 
Gómez, Martínez-de-Haro, Pozo, Gregory, Villagra, Calle \& Veiga, 2009).

Se trata de un cuestionario diseñado para niños entre ocho y 14 años. Se lleva a cabo de manera autoadministrada, y está diseñado para medir actividad física moderada y vigorosa realizada en los últimos siete días, considerando las horas de educación física, actividades extraescolares y tiempo de ocio, tanto durante la semana como el fin de semana. Está formado por 10 ítems, de los que nueve de ellos se utilizan para calcular el nivel de actividad. Cada uno de los ítems tiene una puntuación del uno al cinco, considerando los nueve primeros ítems para el cálculo del resultado global del test, mediante una media aritmética de las puntuaciones obtenidas (M anchola-González, Bagur-Calafat \& Girabent-Farrés, 2017). El ítem 10 tiene como objetivo constatar si el niño encuestado ha estado enfermo 0 ha tenido algún impedimento personal para no realizar su actividad física regular en la última semana. La puntuación final es el resultado de la media aritmética de los primeros nueve ítems. U na puntuación de uno indica un bajo nivel deAF, mientras que una puntuación de cinco indica un alto nivel de AF. N ingún alumno manifestó haber estado enfermo o no haber realizado unaAF similar a su actividad regular. Para el análisis estadístico la muestra se dividió en terciles, clasificándolos en al to, medio y bajo.

Adherencia a la dieta mediterránea

Se utilizó para valorar la adherencia a la DM el test KIDM ED (Serra-Majem, Ribas, N go, O rtega, García, Pérez-Rodrigo \& Aranceta, 2004), un cuestionario de 16 preguntas que deben contestarse de manera afirma tiva/ negativa. Este test consta de cuatro ítems con connotación negativa los cuales si son contestadas de forma positiva se valora con -1 punto (P6-12-14-16), mientras que los ítems con connotación positiva se valoran con +1 punto (P1-2-3-4-5-7-8-9-10-11-13-15). Paraestetest las respuestas negativas no se puntúan. Por tanto, dicho índicetotal oscilaentre-4 y 12, que categorizaunamejor o peor adherencia a la DM : baja calidad $(\leq 3)$; necesita mejorar (4-7); calidad óptima ( $\geq 8$ ).

$\mathrm{N}$ ivel socioeconómico

Se utilizó FAS III (Family Affluence Scale), habiendo sido validado paramedir el NSE de losdiscentes (Hobza, Hamrik, Bucksch \& De Clercq, 2017). Según ValenciaPeris, Devís-Devís y Peiró-Velert (2014), esta medida refleja los recursos materiales de la familia, es decir, los bienes específicos que pueden permitirse sus miembros con los ingresos familiares.

Los ítems se puntúan entre cero y tres puntos, obteniéndose la puntuación total a través de la suma global de estos y con una escala de puntos ordinales, se clasificó el nivel socioeconómico familiar; categorizados en afluencia baja ( 0 - 2), afluencia media ( 3 - 5) y afluencia alta ( $\geq 6$ ) (Moreno-M aldonado, Moreno $\&$ Rivera, 2016).

\section{Análisis estadístico}

Las variables cuantitativas se presentan con la media y la desviación estándar. La normalidad de los datos fue analizadautilizando el test deKolmogorov-Smirnov.Tras comprobar que las variables seguían una distribución no normal se analizaron los datos mediante las pruebas de $U$ de Mann-W hitney para la comparación de dos grupos y Kruskal Wallis en el caso de comparar más de dos grupos. Las correlaciones se realizaron a través del test de Spearman. Para el análisis de los datos, se utilizó el programa estadístico IBM SPSS en su versión 25.0. Se programó el nivel de significación en $p=.05$.

\section{Procedimiento}

En primer lugar se solicitó la colaboración de tres centros educativos de la capital de Granada por conveniencia de los investigadores, a través de una carta informativa confeccionada por el departamento de Didáctica de la Expresión Musical, Plástica y Corporal de la Facultad de Ciencias de la Educación de la U niversidad de Granada. Una vez obtenido el permiso de los centros, se obtuvo el consentimiento informado de los padres o tutores legales y finalmente, se procedió a la real ización de los cuestionarios por parte del alumnado en horario lectivo, en presencia de al menos de uno de los investigadores, garantizando así el uso correcto del instrumento utilizado y resolviendo dudas previas a la aplicación del mismo. Este proceso se llevó a cabo durante los meses de febrero y marzo de 2020. El estudio respetó el acuerdo sobre ética de investigación de Helsinki. El Comité Ético de la U niversidad de Grana da aprobó este estudio.

\section{Resultados}

La Tabla 1 muestra los datos registrados sobre el sexo, adherencia a la DM, y NSE. Del alumnado encuestado, un $57.1 \%$ fueron niños y un $42.9 \%$ niñas. Un $14.3 \%$ de participantes presentó una adherencia a la DM baja, un $65.2 \%$ a una adherencia media y un $20.5 \%$ alta. Atendiendo al NSE, el $12.5 \%$ del alumnado fueron valorados con un nivel bajo, un $44.6 \%$ con un nivel medio y un $42.9 \%$ con un nivel alto. 
LaTabla 2 muestra los valores deAF, adherencia a la DM y NSE según el sexo. En cuanto a los niveles deAF los niños obtuvieron puntuaciones más altas en el PAQ $C$ que las niñas $(2.75 \pm 0.63$ vs $2.31 \pm 0.54)$, siendo estas diferencias estadísticamente significativas $(p=$ .001). En cuanto a la adherencia a la DM y NSE no se encontraron diferencias estadísticamente significativas según el sexo, aunque los niños mostraron puntaciones ligeramente superiores en el test de adherencia a la DM.

La Tabla 3 muestra los valores de adherencia a la DM y NSE según el nivel de AF realizada. Se observó que el alumnado que presenta un mayor NSE, corresponde al tercil alto de AF, siendo estas diferencias estadísticamente significativas ( $p=.001)$, además se puede observar cómo las puntuaciones de AF realizada decrecen conforme disminuyeel NSE $(5.95 \pm 2.08$ frente a $4.73 \pm 1.66$ y $4.45 \pm 1.72$ ).

Aunque no de forma significativa, se pudo observar que los alumnos que obtuvieron una puntuación más baja deAF obtuvieron peores puntuaciones de adherencia a la DM que sus coetáneos, que presentaron una puntuación mayor ( $5.39 \pm 2.11$ vs $6.35 \pm 2.03$ ).

Las correlaciones entre variables se recogen en la Tabla 4. La puntuación obtenida en el PAQ -C correlacionó de manera positiva con la variable de la adherenciaalaDM $(r=.216)$. Además, este correlacionó positivamente con la variable de NSE $(r=.235)$, especialmente en los chicos ( $r=.263)$.

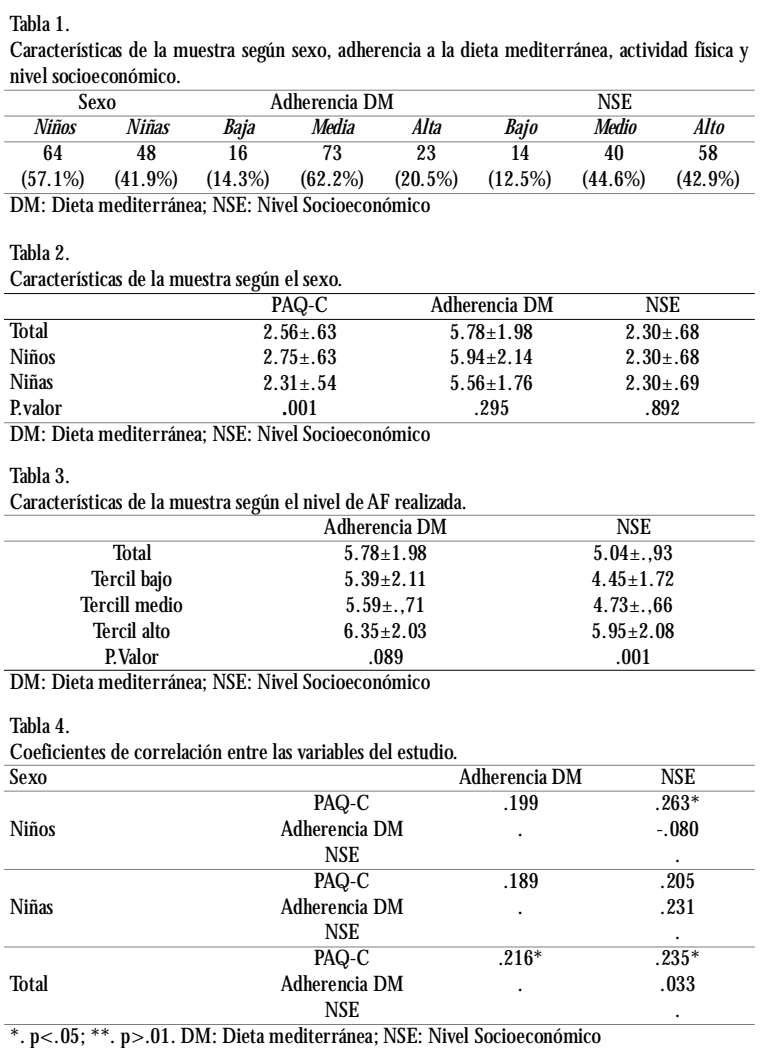

\section{Discusión}

Según esta investigación con 112 escolares de Educación Primaria, ocho de cada 10 estudiantes necesitan mejorar su alimentación, ya que solamente el 20.5\% de los participantes denota una al ta adherencia a la DM. Resultados similares se presentaron en un estudio con 1676 escolares españoles, entre seis y 17 años, ya que solo un $35.7 \%$ se relacionaba con un dieta adecuada (M anzano-Carrasco, Sánchez-Sánchez, H ernándezMartin, Gallardo \& García Unanue, 2020). En la misma línea, se encuentra la investigación de Gil, Cavichiolli \& Lucas (2020) con 370 estudiantes de Educación Primaria (de seis a 13 años), donde manifestaron que solamente el $25.9 \%$ de los participantes mostraban una buena adherencia a la DM. De igual forma, lo presentan Iglesias, Panells \& Molina (2019) en su estudio con 115 niños de 50 y 60 de Educación Primaria, donde los resultados denotaron que la mayoría de los alumnos no cumplían las recomendaciones mínimas y excedían el consumo de ultraprocesados. En contraste a estos porcentajes, se presentan los resultados obtenidos en un estudio con 600 estudiantes del sur de España cuyo porcentaje (58\%) denota que más de la mitad de los participantes expresan tener una alta adherencia a la DM (O nneti, Álvarez-Kurogi \& Castillo-Rodríguez, 2019); estos resultados favorables han de ser consecuentes con la edad del estudio, ya que la población seleccionada, en este caso, cuenta con edades comprendidas entre los 18 y 26 años. En torno a estos años, el sujeto es más independiente y es capaz de desarrollar patrones autónomos en sus cuidados (Gaete, 2015).

En cuanto a la diferencia por sexos en la adherencia a la DM se observó que los niños mostraron puntuaciones ligeramente superiores que las niñas, aunque estos datos no fueran estadísticamente significativos. Resultados totalmente contrarios a los propuestos por Korkmaz y Kebaran (2020) en su estudio con 900 escolares de Educación Primaria entre los seis y nueve años; siendo las niñas (5.2 \pm 2.48$)$, quien presentaba puntua ciones más altas que los niños (4.8 \pm 2.40$)$. En la misma línea, con un total de 515 niños y niñas chilenos, se presenta el estudio de Muros et al. (2017) donde se muestra que las niñas obtienen puntuaciones más al tas que los niños en el KIDM ED ( $6.6 \pm 2$ frente a $5.6 \pm 2.9)$. También Santos, Oliveira, Rodrigues, Barbosa, da Conceicao yVierira (2020) en su investigación con 891 estudiantes portugueses entre nueve y 10 años, el grupo femenino obtiene puntaciones ligeramente superiores que los niños. Esta desigualdad en los resultados ha 
de vincularse a factores externos, como puede ser la zona geográfica en la que se reside y los convivientes; ya que en estas edades, los niños y jóvenes se vinculan y dependen estrechamente de la situación de sus progenitores o tutores legales (Martín, M uóz de Bustillo, Rodríguez \& Pérez, 2008).

Una adherencia alta a la DM ha de utilizarse como herramienta para combatir la obesidad que ocupa a la población actual, valorando otros hábitos de vida saludables, como es el caso de la práctica de AF; ya que son muchas investigaciones las que demuestran los múltiples beneficios que puede atraer a la vida de los escola res (ZuritaO O rtega, U bago-Jiménez, Puertas-M olero, González-Valero, Castro-Sánchez \& Chacón Cuberos, 2018; Dimitri, J oshi \& Jones, 2020). Respecto a los niveles deAF, del mismo modo y en consonancia con un estudio de 11110 niños de diez países europeos (M cM ahon, et al., 2017), se observa que laAF del grupo masculino es más alta que la de del grupo femenino. Datos coincidentes también con el estudio longitudinal de Telford, Telford, O live, Cochrane y Davey (2016) con una muestra de 555 niños de 29 escuel as diferentes entre los ocho y 12 años; el estudio de Castillo, Tomero \& García (2018) compuesto por 392 escolares entre 11 y 17 años y con la investigación de U bago-Jiménez, Chacón-Cuberos, Puertas-Molero \& Ramón-Granizo (2020), centrada en escolares de primaria entre los 10 y 12 años. Estas diferencias pueden estar vinculadas a la influencia de los estereotipos de género, tanto en la sociedad como en la enseñanza (Isorna, Felpeto, Alonso, Gómez \& Rial, 2019).

Aunque no de forma significativa, se pudo observar que los alumnos que obtuvieron una puntuación más baja deAF obtuvieron peores puntuaciones de adherencia a la DM que sus coetáneos, que presentaron una puntuación mayor $(5.39 \pm 2,11$ frente a $6.35 \pm 2.03)$. Siguiendo el mismo patrón en los resultados, un estudio con 200 escolares pertenecientes a la Región de Murcia, con edades comprendidas entre los ocho y 12 años, muestran como los niños con una mayor adherencia a la $D M$ tienen puntuaciones más al tas en la práctica de $A F$ $(9.90 \pm 3.86$ frente a $3.68 \pm 4.18)$ (García \& CarrilloLópez, 2020); mediando además con otros estudios en escolares de primaria donde se manifiesta que a mayor práctica de AF mejor calidad, en general, de la DM (Arriscado, Muros, Zabala \& Dalmau, 2015; J00, Williamson, Vázquez, Fernández \& Bray, 2019) subra yando la correlación positiva hallada en el presente estudio entre la práctica de AF y la adherencia a la DM $(r=.216)$.
Estudios previos han demostrado que un alto NSE se asocia a un aumento considerable de la frecuencia de la AF (Lavin, Bruzzone, Mamondi, González \& Berra, 2015); tal y como se ha observado en los resultados propios, el alumnado con un mayor NSE, corresponde al tercil alto deAF. De la misma forma, se presenta que las puntuaciones de AF disminuyen conforme decrece el NSE; así se confirma en una investigación con 4059 niños de seis años; donde el grupo con un mayor status socioeconómico presenta unas puntuaciones mayores tanto en los hábitos de vida sal udables en general, como en la práctica de AF; por el contrario el grupo con un status socioeconómico menor se relacionó estrechamente con niveles más altos de sedentarismo (Yang-Huang, van Grieken, Wang, Jansen \& Raat, 2020). Esta relación puede deberse, tanto al coste de acceso a prácticas deportivas, siendo una barrera para las familias con menor poder adquisitivo (Fernández-Prieto, Giné-Garriga $\&$ Canet, 2019); como a lainfluencia del nivel educativo de los padres, ya que conforme aumenta el clima educativo de los hogares se incrementa la probabilidad de práctica deportiva (Farinola, Tuñón, Laíño, Marchesich \& Pérez, 2018).

La investigación contó con una serie de limitaciones. Entre ellas, se ha de destacar la utilización del diseño transversal en la investigación, por lo que este estudio no es válido para determinar relaciones causales. Además, hay que mencionar las limitaciones inherentes de precisión en el uso de cuestionarios debido a que los resultados fueron autoreportados por los alumnos. Sin embargo, consideramos que su implicación es mínima debido a que estos cuestionarios han sido previamente validados en poblaciones similares. Aunque el tamaño de la muestra es relativamente grande, la elección de la muestra por conveniencia hace que no se vean representados todos los distritos de la capital de Granada.

Como conclusión, el estudio mostró relaciones significativas entre la práctica de la AF y la adherencia a la $D M$, obteniéndose en ambos casos puntuaciones superiores para el sexo masculino. También se ha de mencionar la necesidad de mejorar la alimentación de los escolares ya que el porcentaje de baja adherencia a la DM, sigue siendo muy elevado. Por otro lado, se reveló que la condición socioeconómica es un factor influyente en la práctica de laAF, siendo el NSE bajo el grupo más desfavorecido en este parámetro. Estos resultados denotan la importancia de fomentar y mejorar la práctica deAF y la adquisición de hábitos saludables, como tener una adherencia alta a la DM, desde edades tempranas. Por lo tanto, es necesaria la implementación de políti- 
cas y programas de intervención, tanto de educación al imentaria como de la práctica de $\mathrm{A} F$, relacionadas con la promoción de la sal ud; que ayuden a la adquisición de hábitos saludables y busquen el equilibrio entre las desigualdades socioeconómicas presentes en el alumnado, tras haber observado la estrecha relación que se da entre el estatus socioeconómico bajo, la inactividad física y los peores hábitos dietéticos.

\section{Referencias}

Arriscado, D. , Muros, J.J., Zabala, M ., \& Dalmau, J.M . (2015). Hábitosde práctica física en escolares: factores influyentes y relaciones con la condiciónfísica. Nutrición H ospitalaria, 31(3),1232-1239. doi:10.3305/ nh. 2015.31.3.8186

Ayechu, A., \& Durà, T. (2009). Dietamediterráneay adolescentes N utrición H ospitalaria, 24(6):759-60. doi:10.3305/ nh.2009.24.6.4567

Castillo, E., Tomero, I. \& \& García, J.A. (2018). Relación entre actividad física, alimentación y familia en edad escolar. Retos, (34), 85-88. doi:10.47197/ retos. v0i34.52782

Díaz-Acosta, R., Shiba-M atsumoto,A.R. . \& Gutiérrez, J.P. (2015). M ediciónsimplificadadel nivel socioeconómico en encuestasbreves: propuesta a partir de acceso abienesy servicios. Salud Pública de M éxico, 57(4), 298-303. doi:10.21149/ spm.v57i4. 7572

Dimitri, P., Joshi, K. \& \& Jones, N. (2020). M ovingmore: physical activity and itspositive effectson longterm conditionsin children and young people. Archives of Disease in Childhood, 105(11), 1035-1040. doi:10.1136/ archdischild-2019-318017

Farajian, P., Risvas, G., Karasouli, K., Pounis, G. D., Kastorini CM , ... DB, ZampelasA. (2011).Very high childhood obesity prevalenceand low adherence rates to the M editerranean diet in Greek children: the GRECO study. Atherosclerosis, 217(2), 525-30. doi:10.1016/ j. atherosclerosis. 2011.04.003

Farinola, M.G., Tuñón, I., Láño,F., M archesich, M ., \& Pérez,M . (2018). Perfil socioeducativo yeconómico de deportistasadolescentesdeélite argentinos. Retos, (34), 172-176. doi:10.47197/ retos. v0i34. 60072

Fernández-Prieto, l. , GinéGarriga, M ., \& Canet, 0. Barrerasy motivaciones percibidas por adolescentes en relación con la actividad física. Estudio cualitativo através degruposdediscusión. Revista Española de Salud Pública, 93,1-12. Recuperado de: http:/ / scielo. isciii.es/ scielo. php?script=sci_abstract\&pid=S113557272019000100049

Gaete,V. (2015). Desarrollo psicosocial del adolescente. Revista Chilena de Pediatía, 86(6), 436-43. doi:10.1016/ j.rchipe. 2015.07.005

Garcia, M., \& Carrillo-Lopez, P. . . (2020). Levels of Physical Activity and Q uality of Diet in Primary School Students. Revista Iberoamericana de Ciencias de la Actividad Física y el Deporte, 9(2), 16-31. doi:10.24310/ riccafd. 2020.v9i2. 7155

Garrido-Fernández,A.García-Padilla, F.M., Sánchez-Ramos,_.L., GómezSalgado, J.,TravéG onzález, G. \& Sosa-Cordobés, E. (2020). Alimentosconsumidospor estudiantes desecundariaduranteel díaescolar. Nutrients, 12(2), 1-18. doi:10.3390/ nu12020485

Gil, J.F.L., Cavichiolli, F.R, \& Lucas, J.L.Y. (2020). Programas de intervención paralapromoción dehábitosalimenticiossaludablesen escolaresespañoles practicantes deEducación Física: U narevisión sistemática. Retos, (37),786-792. doi:10.47197/ retos. v37i37.69931

González,A., García, M. F., Martos, l., Liébana, J.L., \& M elo, J.A. (2015). Prácticasalimentarias del alumnado de secundariadurantelajornada escolar enAndalucíazsociadasalaofertadel entorno. Nutrición H ospi- talaria, 31(4),1853-1862. doi:10.6018/ eglobal.16.2.239881

Guthol, R., Stevens, G. A., Riley, L. M., \& Bull, F.C. (2020). Global trends in insufficient physical activity among adolescents: apooled analysis of 298 population-based surveys with 1.6 millions participants. Lancet Child Adolescent Health, 4(1),23-35. doi: 10.1016/ S23524642(19)30323-2

Herrera-Mora, D. B., Munar-Torres,Y.E., MolinaAchury, N.J. , \& RobayoTorres,A.L. (2019). Child development and socioeconomic status. Review article. Revista de la Facultad de M edicina, 67(1), 145-52. doi:10.15446/ revfacmed.v67n1.66645

Hobza, V., Hamrik, Z., Bucksch, J. \& DeClercq, Bart. (2017).TheFamily AffluenceScaleasan indicator for socioeconomic Status: validation on regional incomedifferencesintheCzech Republic. International Journal of Environmental Research and PublicH ealth, 14(1540), 1-9. doi:10.3390/ ijerph14121540

Iglesias, A., Planells, E. \& Molina, J. (2019). Prevalencia de sobrepeso y obesidad, hábitos alimentarios y actividad física y su relación con el rendimiento académico. Retos, (36), 167-173. doi:10.47197/ retos. v36i36. 66873

Instituto Nacional deEstadística(2017). Consumo defrutas, verduras, ensaladas y hortalizas (2017) [fichero de datos]. Recuperado de: https:/ / www.ine.es/ up/ Khe7Cnld

Instituto Nacional de Estadística(2017). Sedentarismo según gruposde edad (2017) [fichero dedatos]. Recuperado de: https:/ / www. ine.es/ up/ gqEK dIYj

Isorna, M. ., Fel peto, M.,Alonso, D., Gómez, P., \& Rial,A. (2019). Mujer y piragua: estudio de lasvariablesmoduladores del abandono deportivo delasmujerespiragüistasen modalidadesolímpicas. Retos, 35(1), 320-325. doi:10.47197/ retos. v0i35.66800

Jo0, J., W illiamson, S.A., Vazquez, A.I., Fernandez, J.R., \& Bray, M.S. (2019). Theinfluenceof 15-week exercisetraining on dietary patterns amongyoungadults. International Journal of 0 besity, 43(9),1681-1690. doi:10.1038/ s41366-018-0299-3

Kontogianni, M., Vidra, N., Farmaki, A.E., Koinaki, S., Belogianni, ... Yannakoulia, M. (2018).AdherenceRatesto theM editerranean Diet Are Low in a Representative Sample of Greek Children and Adolescents. Journal of N utrition, 138(10), 1951-1956. doi:10.1093/ jn/ 138.10.1951

Korkmaz, G.0., \& Kabaran, S. (2020). Protectiveeffectsof aM editerraneanlike dietary patern on obesity, abdominal obesity and large neck circumferencein acohort of Turkish children aged 6-9 years Asia Pacific Journal of Clinical Nutrition, 29(2),363-371. doi:10.6133/ apjcn.202007_29(2).0019

Lahoz, M. . Gracia, F. , \& Hervás, J. (2010).Sin vino. .. . ¿estásseguro, cora zón? Delatrilogía mediterráneaala paradojafrancesa. Enfermería en Cardiología, 50(2.0 cuatrimestre), 22-27. https:// www. enfermeriaencardiologia.com/ revista/ autores/ camanessalva dor-a

Lavin, J., Bruzzone, F., M amondi,V., González, E., \& Berra, S. (2015).Actividad Físicay comportamientossedentariosdeescolaresdeCórdoba en su tiempo librey en clase deEducación Física. Revista Argentina de Salud, 6(24), 15-21. http:/ / hdl. handle.net/ 11336/ 58204

López, G.F., Ahmed, D., López, F.J., Dízz, L., \& Díaz,A. (2016). Nivel de actividad física habitual en escolares de 8-9 años de España e India. Movimiento Humanoy Salud, 15(2). doi:10.15359/ mhs. 12-2.3

Manchola-González, J., Bagur-Calafat, C. \& Girabent-Farrés, M. (2017). Fiabilidad de la versión española del cuestionario de actividad física PAQ -C. Revista Internacional deM edicina y CienciasdelaActividad Física del Deporte, 17(65), 139-152. doi:10.15366/ rimcafd2017.65.010

Manzano-Carrasco, S., Luis, J., Sanchez-Sanchez, J., Hernandez-M artin, 
A., Gallardo, L., \& GarciaU nanue, J. (2020).WeightStatus,Adherence to theM editerranean Diet, and Physical Fitnessin Spanish Children andAdolescents: TheActive Health Study. Nutrients, 12(1680), 1-14. doi:10.3390/ nu12061680

Martín, E., MuñozdeBustillo, M.C., Rodríguez,T., \& Pérez,Y, (2008). De la residencia a la escuela: la integración social de los menores en acogimiento residencial con el grupo deigualesen el contexto escolar. Psicothema, 20(3):376-382. http:/ / www. redalyc.org/ articul 0.0 arid $=72720306$

Martínez-Gómez, D., Martínez-de-Haro,V., Pozo,T.,Welk, G.J.,Villagra, A., Calle, M., Marcos,A.\&Veiga, O.L. (2009). Fiabilidad y validezde cuestionario de actividad física PAQ -A en adolescentes españoles. Revista Española deSalud Pública, 83(3), 427-739.

McMahon, E.M, Corcoran P, O'Regan G, Keeley $H$, Cannon,... Wasserman, D. (2017). Physical activity in European adolescentsand associationswith anxiety, depression and well-being European Child \&Adolescent Psychiatry, 26 (1),111-122. doi:10.1007/ s00787-016-0875-9

Moreno-M aldonado, C., Moreno, C., \& Rivera, F. (2016). Indicadores paradetectar yevaluar el impacto delasdesigualdadessocioeconómicas en losestilosdeviday lasalud delosadolescentes ApuntesdePsicología, 34(2-3), 177-188. http:/ / hdl. handle.net/ 10486/ 677766

Muros, J.J., Cofre-Bolados, C., Arriscado, D., Zurita, F., \& Knox, E. (2017). Mediterranean diet adherence is associated with lifestyle, physical fitness, and menta wellnessamong10-y-oldsinChile. Nutrition, (35),87-92. doi:10.1016/ j.nut.2016.11.002

O rganización Mundial de la Salud. (2017). 10 datos sobre la actividad física. Ginebra: OMS. https:/ / www. who.int/ features/ factfiles/ p h y s i c a l a c t i v i t y / e s / \# : : text $=$ La\% 20actividad $\% 20 f \%$ C 3\% AD sica $\% 20$ red uce $\% 20$ el $\% 20$ riesgo $\% 20$ de $\% 20$ cardiopat $\%$ C 3\%AD as\%20coronarias\%20y,y\%20el\%20control\%20del\%20peso

O rganización M undial delaSalud. (2018). Actividad Física Ginebra: O MS. https:/ / www. who. int/ es/ news-room/ fact-sheets/ detail/ physicalactivity

O rganización M undial delaSalud. (2019). U n nuevo estudio dirigido por la OMS indica que la mayoría de adolescentes del mundo no realiza suficiente actividadfísica, y queeso poneen peligrosu salud actual y futura. Ginebra: $0 \mathrm{MS}$. https:/ / www. who. int/ es/ news/ item/ 22-11-2019-new-who-ledstudy-says-majority-of-adolescents-worldwide-are-not-sufficientlyphysically-active-putting-their-current-and-future-health-at-risk

O netti,W., Álvarez-Kurogi, L., Castillo-Rodríguez,A., O netti,W., ÁlvarezKurogi, L., \& Castillo-Rodríguez,A. (2019).Adherenciaal patrón de dietamediterráneay autoconcepto en adolescentes. Nutrición H ospitalaria, 36(3),658-64. doi:10.20960/ nh.02214

Patton, G.C., \&Viner, R. (2007). Pubertal transitions in health. Lancet, 369(9567),1130-1139. doi:10.1016/ S0140-6736(07)60366-3

Rees, K., Takeda, A., Martin, N., Ellis, L., W ijesekara, D., ... Stranges, S. (2019). Mediterranean-style diet for the primary and secondary prevention of cardiovascular disease. CochraneSystematic Review,3(3),1126. doi:10.1002/ 14651858.CD009825. pub3

Rodríguez, J., Iglesias, A., \& Molina, J. (2020). Evaluación de actividad física, laadherenciaaladietamediterráneay el comportamiento y su relación con lacalidad devidaen estudiantes deEducación Primaria. Retos, (38), 129-136. doi:10.47197/ retos. v38i38. 73921

Roman, B. , Serra, L., Ribas, L. , Pérez-Rodrigo, C. , \&A ranceta, J. (2006). Actividad físicaen lapoblación infantil yjuvenil españolaen el tiempo libre. Estudio enKid (1998-2000). Apunts, 41(151),86-94. doi:10.1016/ S1886-6581(06)70016-0

Sánchez, R., Reyes, H., \& González, M.A. (2014). Preferenciasalimentarias yestado denutrición en niñosescolaresdelaCiudad deM éxico. Boletín Médico del Hospital Infantil de México, 71(6),358-66. doi:10.1016/ j.bmhimx.2014.12.002

Sánchez-Urrea, A. \& Izquierdo,T. (2021). Factoressocioeconómicosque influyen en la salud nutricional y actividad física de escolares. R etos, (40), 95-108. doi:10.47197/ retos.v1i40.81106

Santos, G. F., O liveira, S. , Rodrigues,A.C., Barbosa, T.D. , daConceicao, A.P., \&Vieira, A.R. (2020)Adherence to the mediterranean diet in elementary school children (1st cycle). Revista Paulatista de Pediatría, 39,1-9. doi:10.1590/ 1984-0462/ 2021/39/ 2019259

Serra, L. , Ribas, L., Pérez, C., Román, B. \&Aranceta, J. (2003). Hábitos alimentariosy consumo dealimentosen lapoblación infantil yjuvenil española(1998-2000):Variablessocioeconómicasy geográficas M ediànaClínica, 121(4), 126-31. doi:10.1016/ S0025-7753(03)73879-2

Serra-Majem, L., Ribas, L., Ngo, J. , O rtega, R.M. ., GarcíaA, ... A ranceta, J. (2004).Food, youthand themediterranean dietin Span. Development of kidmed, mediterranean dietquality index in children and adolescents. PublicH ealth N utrition, 7, 931-935. doi: 10.1079/ PHN 2004556

Tapia, A. (2019). Diferencias en los niveles de actividad física, grado de adherenciaaladietamediterráneay autoconcepto físico en adolescentes en función del sexo. Retos, (36),185-192. doi:10.47197/ retos.v36i36. 67130

Telford, R.M., Telford, R.D., O live, L.S., Cochrane, T., \& Davey, R. (2016).W hy aregirl lessphysically activethan boys? Findingsfrom the look longitudinal study. Plos One,11(3),1-11. doi:10.1371/ journal. pone.0150041

U bago-Jiménez, J.L., Chacón-Cuberos, R., PuertasM olero,P., \& RamírezGranizo, I.A. (2020). Influenciadeladietay hábitosfísico-saludables en escolares. Revista Iberoamericana de Ciencias dela Actividad Física y el Deporte, 9 (1), 106-113. doi:10.24310/ riccafd.2020. v9i1.8306

ValenciaPeris, A., DevísDevís,J., \& Peiró-Velert, C. (2014). El uso sedentario demediostecnológicosdepantalla: perfil sociodemográfico de los adolescentes españoles (Sedentary use of screen-media: Sociodemographic profileof Spanishadolescents). Retos, (26), 21-26. doi: 10.47197/ retos. v0i26.34389

Watson, A. , Timperio, A., Brown, H., Best, K. , \& Hesketh, K.D. (2017), Effect of classroom-based physical activity interventions on academic and physical activity outcomes: asystematic review and meta-analysis. International Journal of Behavioral Activity, 14 (114), 1-24. doi:10.1186/ s12966-017-0569-9

Yang-H uang, J., van Grieken,A.,Wang, L., Jansen, W.\&, Raat, H. (2020). Clustering of Sedentary Behaviours, Physical Activity, and EnergyDenseFood IntzeinSix-Year-O Id Children:AssocidionswithFamily Socioeconomic Status. N utrition,12(1722),1-13. doi:10.3390/ nu12061722

Yannakoulia, M. , Lykou,A., K astorini, C.M., Papasaranti, E.S. , Petralias, A., Veloudaki, A. ... DIATROFI Program Research Team. Socioeconomicand lifestyleparametersassociated with diet quality of children and adolescents using classification and regression tree analysis: The DIATROFI study. PublicH ealth Nutrition, 19 (2),339-47. doi:10.1017| S136898001500110X

ZuritaOO rtega, F., U bago-Jiménez, J.L., Puertas-M olero, P., GonzálezValero,G., Castro-Sánchez, M., \& Chacón-Cuberos, R. (2018). Niveles de actividad física en alumnado de Educación Primaria de la provincia de Granada. Retos, (34),218-221. doi:10.47197/ retos.v0i34.60098 